\title{
Predictors of Psychiatric Disorders or uses of Psychotropic Drugs in Patients with Confirmed COVID-19 During Treatment in a Hospital : A Retrospective Chart Review Running title : Mental health of COVID-19 patients
}

Jang Rae Kim

National Medical Center

Myung Hwa Han

National Medical Center

So Hee Lee ( $\nabla$ sohee.lee@nmc.or.kr)

National Medical Center https://orcid.org/0000-0002-9005-3207

Research article

Keywords: Coronavirus Disease-19, psychiatric disorders, psychotropic drugs, acute phase, isolation wards

Posted Date: August 21st, 2020

DOI: https://doi.org/10.21203/rs.3.rs-59380/v1

License: (a) (1) This work is licensed under a Creative Commons Attribution 4.0 International License. Read Full License 


\section{Abstract}

\section{Background}

This study aims to examine the psychiatric diagnoses, psychotropic drug use, and related factors in patients with coronavirus disease-19 (COVID-19) during their acute treatment phase.

\section{Methods}

We performed a retrospective chart review of patients admitted to the isolation ward at a nationally designated hospital during the COVID-19 outbreak. All the COVID-19 patients admitted to the hospital were assigned to psychiatrists by consultation in order to monitor their mental health during their hospital stay. We analyzed the medical records and self-reported psychological tests of 80 out of 112 patients.

\section{Results}

On admission, $16.3 \%$ of the subjects had self-reported depression and $6.3 \%$ had post-traumatic stress disorder (PTSD). Twenty-five patients (31.3\%) had current diagnoses of mental disorders. These disorders consisted of nonorganic insomnia, acute stress reaction, panic disorder, and depressive episodes. Nineteen patients (23.8\%) were prescribed psychotropic drugs, including trazodone, alprazolam, clonazepam, and zolpidem. Factors associated with these psychiatric diagnoses included self-reported PTSD on the day of admission and having a spouse. Previous psychiatric history and age of 40 years or more were associated with the use of psychotropic drugs.

\section{Conclusion}

Mental health services rendered to quarantined COVID-19 patients undergoing an acute treatment phase and self-reported PTSD on the day of admission might be useful in predicting mental health problems during hospital days.

\section{Background}

The outbreak of COVID-19, caused by the novel severe acute respiratory syndrome coronavirus 2 (SARSCoV-2), had an enormous impact on medical, psychological, and social issues globally. Until June 27, 2020, at least 12,653 cases of COVID-19 had been confirmed in South Korea [10], with 282 known deaths, representing a fatality rate of approximately $2.2 \%$. The estimated fatality rate of COVID-19 indicates that its clinical presentation would be milder than that of other EIDs (emerging infectious diseases), about $36 \%$ for MERS [17] and about $10 \%$ for SARS [9].

However, details of COVID-19's clinical characteristics and epidemiological features have not yet been fully identified. Older patients and those with an underlying medical disease are less likely to survive [20], possibly because of a weakened immune system that permits a faster progression of viral infection. Therefore, a confirmed diagnosis of COVID-19 can cause anxiety or panic in patients who must be 
isolated in rooms for weeks or months. This can be a very stressful event. Therefore, although COVID-19 is a physical illness, this pandemic also raises mental health issues for health care workers, people under quarantine, people admitted to isolation wards, as well as those who have recovered from this infection [3].

During their treatment for EIDs, quarantined patients reported feeling psychiatric symptoms. A systematic review revealed that during their acute illness, patients admitted to the hospital for SARS or MERS suffered such psychological problems as depressed mood, anxiety, and insomnia [13]. In addition, they were also noted to have confusion and impaired memory.

Some survivors of EIDs are known to have suffered from mental health problems for years. Various forms of psychological distress were prevalent among Ebola survivors, including depression, anxiety, anger, grief, guilt, flashbacks, sadness, worthlessness, substance addiction, suicidal tendencies, and selfstigmatization [5]. The meta-analysis [13] indicated that in the post-illness stage of SARS and MERS, the point prevalence of PTSD was $32.2 \%$, that of depression was $14.9 \%$, and that of anxiety disorder was $14.8 \%$.

When data for patients with COVID-19 was examined, there were cases of such neurological problem as delirium, dysexecutive syndrome, hypoxic encephalopathy, and encephalitis [13]. Psychological morbidities, including PTSD, anxiety, and depression, were reported to be common in COVID-19 patients [12]. Additionally, immune function changes correlated with self-reported depression during short-term follow-up of COVID-19 patients following discharge from the hospital [19].

However, the psychiatric features and related factors of COVID-19 patients undergoing treatment have not yet been sufficiently investigated. Therefore, the purposes of this study are (1) to examine the prevalence of current psychiatric disorders and psychotropic drug use in the acute phase of COVID-19 treatment, and (2) to know their risk factors.

\section{Methods}

\section{Sample}

We reviewed the medical records of all patients with confirmed COVID-19 admitted to the isolation wards at the National Medical Center (NMC) between February 1 and April 30, 2020. We defined a patient with COVID-19 as one who had been found to be positive on real-time reverse transcription polymerase chain reaction (RT-PCR), conducted and confirmed by our laboratory medical staff. The South Korean government has designated the NMC to be the central hospital for infectious diseases since the outbreak of Middle East respiratory syndrome (MERS). It has concentrated its resources on managing and treating COVID-19 patients following the outbreak of COVID-19 and dedicated all its wards with negative pressured rooms to these patients.

\section{Consultation strategy}


All the COVID-19 patients admitted to the NMC were assigned to psychiatrists by consultation in order to monitor their mental health during their hospital stay. We carried out psychiatric interventions if needed. Reflecting our experience with MERS patients in 2015 [7], we offered two modes of mental health services to all COVID-19 inpatients starting on their first hospital day

1. If eligible, we gave the patients psychological tests with self-report questionnaires within a few days following admission.

2. We offered daily telephone calls during psychiatric rounds to check for any disturbances of daily living. Further, we conducted face-to-face interviews at the patient's request or when acute psychiatric symptoms emerged.

\section{Measures}

Information concerning demographic characteristics, previous history of medical illness or psychiatric symptoms, presence of pneumonia, results of psychological assessments, psychiatric diagnosis and psychotropic drugs prescribed during quarantined in the hospital.

The psychological tests consist of the Patient Health Questionnaire-9 (PHQ-9) and the PTSD Checklist for DSM-5 (PCL-5). We distributed the short versions of the Patient Health Questionnaire-2 (PHQ-2) and the primary Care PTSD (PC-PTSD) to elderly patients who were 60 years old or older.

We used the PHQ-9 to monitor the severity of depression [8]. The questionnaire had nine items based on the criteria for depression in the Diagnostic and Statistical Manual of Mental Disorders, fourth edition (DSM-IV). The patient could rate each question from 0 to 3 to provide a severity score ranging from 0 to 27. We defined depression severity as being no depression (1-4), mild depression (5-9), moderate depression (10-14), moderately severe depression (15-19), and severe depression (20-27). We considered significant depression to be present when the total score was 10 or higher. The Korean version of the PHQ-9 has been shown to be a reliable and valid tool for the screening and assessment of depressed patients [1]. The Cronbach's alpha coefficient was 0.88 .

The PCL-5 is a self-rated inventory developed for screening PTSD symptoms based on DSM- 5 criteria [16]. It includes 20 items, and each item has five response options with scores ranging from 0 to 4 . The total score of the PCL-5 scale ranges from 0 to 80 . A score of 33 is used as the cut-off for a PTSD diagnosis. The Cronbach's alpha coefficient was 0.93 .

The PHQ-2 is a briefer measure for depression used to shorten test time [4]. The questionnaire consists of three items from PHQ-9, each scored from 0 to 3. The PHQ-2 has been translated and validated in Korean and has shown good validity and reliability, with its optimal cut-off score of 3 for depression.

The PC-PTSD is a timesaving instrument for screening PTSD symptoms, having five items requiring yes or no responses. It is identical to the PC-PTSD, with the exception of the revised trauma exposure stem and the addition of a fifth item [6]. The Korean version of the PC-PTSD has shown a good internal 
consistency ( $a=0.872)$, test-retest reliability ( $r=0.89)$, and concurrent validity $(r=0.81)$. We identified a score of 3 as being the threshold for clinically significant posttraumatic stress disorder (PTSD) symptoms. 
Table 1

Sociodemographic characteristics of the study population

\begin{tabular}{|c|c|}
\hline \multirow[t]{2}{*}{ Variable } & Total $\mathbf{N}=\mathbf{8 0}$ \\
\hline & $\mathrm{N}(\%)$ or Mean \pm SD \\
\hline \multicolumn{2}{|l|}{ Gender } \\
\hline Male & $53(66.3)$ \\
\hline Female & $27(33.8)$ \\
\hline \multicolumn{2}{|l|}{ Age (years) } \\
\hline Total & $40.8 \pm 17.9$ \\
\hline$\leq 29$ & $31(38.8)$ \\
\hline $30-39$ & $15(18.8)$ \\
\hline $40-49$ & $10(12.5)$ \\
\hline $50-59$ & $6(7.5)$ \\
\hline$\geq 60$ & $18(22.5)$ \\
\hline \multicolumn{2}{|l|}{ Marital status } \\
\hline Married & $35(43.8)$ \\
\hline Single & $42(52.5)$ \\
\hline Divorced or separated & $3(3.8)$ \\
\hline \multicolumn{2}{|l|}{ Education } \\
\hline$\leq$ Middle school & $6(7.5)$ \\
\hline High school & $40(50.0)$ \\
\hline$\geq$ University & $34(42.5)$ \\
\hline \multicolumn{2}{|l|}{ Occupation } \\
\hline Worker & $33(41.4)$ \\
\hline Non-worker & $47(58.8)$ \\
\hline \multicolumn{2}{|l|}{ Religion } \\
\hline Yes & $44(55.1)$ \\
\hline No & $36(45.0)$ \\
\hline SD, standard deviation & \\
\hline
\end{tabular}


Table 2

Clinical and psychological characteristics of the study population

\section{Variable}

Yes

No

Previous psychiatric history

Yes

No

Presence of pneumonia

Yes

No

Depression

(PHQ-2) †

(PHQ-9) ††

Yes

No

PTSD

(PC-PTSD) †

(PCL-5) ††

Yes

No

\section{PTSD}

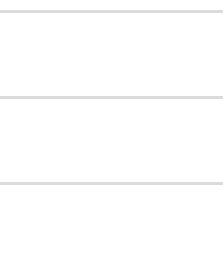

Total $\mathbf{N}=\mathbf{8 0}$

$\mathrm{N}(\%)$ or Mean \pm SD
$30(37.5)$

$50(62.5)$

8(10.0)

72(90.0)
$55(68.8)$

25(31.3) Previous psychiatric history, previous experiences of psychiatric symptoms; PHQ-2, Patient Health
Questionnaire-2; PHQ-9, Patient Health Questionnaire-9;

PC-PTSD, Primary Care PTSD Screen; PCL-5, Posttraumatic Stress Disorder Check List-5.

tn $=14,+t n=66$ 
Table 3

Psychiatric manifestations of COVID-19 patients during admission in a COVID-19 hospital $(\mathrm{N}=80)$

\section{Category}

\section{Frequency}

N $\quad \% \dagger$

Current diagnosis of mental disorders

Depressive episode

Panic disorder

Acute stress reaction

Nonorganic insomnia

Use of psychotropic drug

Quetiapine

Aripiprazole

Escitalopram

Trazodone

Duloxetine

Fluoxetine

Valproate

Lorazepam

Clonazepam

Alprazolam

Zolpidem

2

6

7

11

(26 cases of 25 persons)

$\begin{array}{ll}2 & 7.7\end{array}$

23.1

26.9

42.3
7.9

5.3

5.3

18.4

2.6

2.6

2.6

4

10.5

5

13.2

7

5

18.4

13.2

(38 cases of 19 persons)

COVID-19, Coronavirus disease-19.

tPercentage of the number of cases to the total number of cases in COVID-19 patients who were diagnosed with mental disorders or received psychotropic drugs 
Table 4

Estimated odds ratio for psychiatric manifestations based on possibly related variables in COVID-19 patients

\section{Current diagnosis of mental disorders}

Unadjusted OR

Age, year
$\geq 40$
$3.20(1.21-8.46)^{\star}$

Having a spouse

Yes

$3.78(1.41-10.13)^{\star \star}$

Previous psychiatric history

Yes

Depression

Yes

$4.36(1.26-15.07) \star$

PTSD

Yes

$9.63(1.02-91.05) *$

Previous psychiatric history, previous experiences of psychiatric symptoms ; OR, odds ratio; COVID-19, Coronavirus disease; $\mathrm{Cl}$, confidence interval; Depression, depression scale positive on admission; PTSD, posttraumatic stress disorder scale positive on admission.

$\star P<0.05, * \star P<0.01, * * * p<0.001$

\section{Ethical statement}

The study was approved by the Institutional Review Board for Human Subjects at the NMC in Seoul, S. Korea (NMC-2006-005), and written informed consent was obtained from each patient.

\section{Statistical analysis}

We present the sociodemographic characteristics, COVID-19-related clinical characteristics, as well as the participants' psychiatric clinical information as both numerical values and as percentages. We divided the 80 eligible patients into two groups, according to whether they had any diagnoses of mental disorders or if they had been prescribed any psychotropic medications.

We conducted independent t-tests to compare the PHQ-2/PHQ-9 and the PC-PTSD/PCL-5 scores between the two groups. We used univariate analysis to identify potential factors associated with the diagnosis of mental disorders and the use of psychiatric medications in COVID-19 patients. We performed a stepwise multivariate logistic regression analysis $(P<.05)$ on the variables identified as significant in the univariate analysis. We analyzed all data with the SPSS for Windows software, version 20.0 (IBM Corp.; Armonk, NY, 
USA) except for the logistic regression analysis, which we performed with SAS version 9.4 (SAS Institute Inc.; Cary, NC, USA).

Table 4 Estimated odds ratio for psychiatric manifestations based on possibly related variables in COVID-19 patients

Current diagnosis of mental disorders

Unadjusted OR

Age, year
$\geq 40$
$3.20(1.21-8.46) *$

Having a spouse

Yes $\quad 3.78(1.41-10.13)^{\star \star}$

Previous psychiatric history

Yes

Depression

Yes $\quad 4.36(1.26-15.07)^{\star}$

PTSD

Yes 9.63(1.02-91.05)*

Previous psychiatric history, previous experiences of psychiatric symptoms ; OR, odds ratio; COVID-19, Coronavirus disease; $\mathrm{Cl}$, confidence interval; Depression, depression scale positive on admission; PTSD, posttraumatic stress disorder scale positive on admission.

${ }^{*} \mathrm{P}<0.05,{ }^{*} \mathrm{P}<0.01,{ }^{* * *} \mathrm{p}<0.001$

\section{Results}

There was a total of 112 confirmed COVID-19 patients who had received treatment in the isolation wards at the central hospital for infectious diseases in South Kores. In our study, we excluded those patients with decreased consciousness, psychoses (schizophrenia, bipolar disorders), or neurocognitive disorders (dementia, mental retardation) from psychological assessment. The diagnostic distribution of those patients that we excluded from the test $(n=22)$ were: $19.2 \%$ with Alzheimer's dementia, $3.8 \%$ with unspecified dementia, $7.7 \%$ with other mental disorders due to brain damage, $3.8 \%$ with mental and behavioral disorders caused by alcohol use, $50 \%$ with schizophrenia, and $11.5 \%$ with mental retardation,

We evaluated the remaining 80 patients with self-reported questionnaires. In our study, we included more male $(n=53,66.3 \%)$ than female patients, and $57 \%$ of the subjects were in their 20 s to 30 s (mean age: 40.8, standard deviation [SD]: 17.9) (Table 1). Of those 80 patients, more than two thirds had pneumonia $(n=55,68.8 \%), 37.5 \%$ had underlying medical disease, and $10.0 \%$ had a history of psychiatric symptoms 
prior to the COVID-19 infection. Another $6.3 \%$ had panic disorders, $2 \%$ had depressive episodes, and $1.3 \%$ had nonorganic insomnia (Table 2). A few days following admission, $16.3 \%$ of the subjects reported clinically significant symptoms of depression (based on PHQ-9 cut off scores of 10 or PHQ-2 cut off scores of 3 ), and $6.3 \%$ of these patients were positive on the PTSD scale (based on PCL-5 cut off scores of 33 or PC-PTSD cut off scores of 3$)$. The mean total score on PHQ-2 was 0.79 (SD = 1.19), $5.15(\mathrm{SD}=$ 5.55) on PHQ-9, whereas the mean total score on PC-PTSD was $0.86(S D=1.29), 7.59(S D=10.60)$ on PCL-5.

Overall, 31.3\% (25/80) of the study population had experienced psychiatric symptoms and were diagnosed with psychiatric disorders, and more than half of them (76\%) were prescribed psychotropic drugs during treatment of COVID-19 (Table 3). The total number of psychiatric diagnoses upon discharge was 26 , with an average of 1.04 recorded per patient (26 diagnoses/25 persons). Likewise, we prescribed 38 psychotropic medications, with an average of 2.0 recorded per patient (38 cases/19 persons) during admission in a COVID-19 hospital. The diagnoses of mental disorders were $42.3 \%$ with nonorganic insomnia, $26.9 \%$ with acute stress reaction; $21.3 \%$ with panic disorder, and $7.7 \%$ with depressive episodes. The four most prescribed psychotropic drugs were trazodone (18.4\%), alprazolam (18.4\%), clonazepam (13.2\%), and zolpidem (13.2\%).

We performed univariate and multivariate logistic regression analysis to identify the risk factors associated with the psychiatric diagnoses and the use of psychotropic medication during hospitalization. The univariate analysis revealed that several factors were significantly associated with psychiatric diagnoses at discharge $(P<.02$ and $P<.05)$, including age, having a spouse, self-reported depression, or PTSD on admission. Significantly associated with psychotropic drug use were age, previous experiences with psychiatric symptoms, self-reported depression, and PTSD on admission. Neither gender, education, nor religion was associated with the outcome variables. Also, clinical information, such as the absence of previous medical diseases or pneumonia, was associated with these outcomes.

The multivariate analysis confirmed that having a spouse (aOR: $4.40,95 \% \mathrm{Cl}: 1.53-12.48, \mathrm{P}<.01$ ) or psychological tests on admission showing PTSD positivity (aOR: 13.28, 95\% Cl: 1.26-139.94, p<.05) was predictive of having a diagnosis of mental disorders during a COVID-19 admission. Age (aOR: 3.39, 95\% Cl: 1.03-11.10, $\mathrm{P}<.05$ ) and previous experiences of psychiatric symptoms (aOR: 15.17, 95\% Cl: 2.53-90.98, $p<.001$ ) could be relevant factors associated with receiving psychotropic medications (Table 4).

\section{Discussion}

Our results showed that $31.3 \%$ of subjects with confirmed COVID-19 who could answer self-reported psychological tests received diagnoses of mental disorders, including nonorganic insomnia, acute stress reaction, panic disorder, and depressive episode during admission. We found that the previous experiences of psychiatric symptoms predicted the use of psychotropic agents during admission in isolation wards. These results supported the need for intervention by mental health professionals for patients admitted to EID treatment hospitals [3]. These findings are similar to those of previous studies 
that showed among patients hospitalized for SARS or MERS with acute illness, common psychiatric symptoms included depressed mood, anxiety, and insomnia. Patients with confirmed COVID-19 may fear the consequences of infection with this potentially fatal new virus [18], and those in quarantine might experience boredom, loneliness, associated stigma, and anger. Furthermore, symptoms of the infection, such as fever, hypoxia, and cough, as well as adverse effects of treatment, such as corticosteroid-caused insomnia, could lead to worsening anxiety and mental distress. Moreover, the COVID-19 outbreak inevitably affected many of those patients with chronic psychotic disorders or dementia. These patients needed active management by psychiatrists, although we had excluded them from the study sample because they were not appropriate candidates for evaluation with self-reported scales. Therefore, treatment in isolation wards may precipitate new psychiatric symptoms or aggravate existing conditions in people without mental illness [2].

On admission to a COVID-19 hospital, $16.3 \%$ of our subjects reported depression (PHQ-9 $\geq 10$, or PHQ-2 $\geq 3$ ) and $6.3 \%$ reported PTSD symptoms (PCL-5 $\geq 33$, or PC-PTSD $\geq 3$ ). These results suggest the possibility that psychological problems in COVID-19 survivors might be relatively lower than MERS survivors. In a previous study of South Korea [11], at 12 months post-MERS, $42.9 \%$ of survivors reported PTSD (IES-R-K $\geq 25$ ) and $27.0 \%$ reported depression (PHQ-9 $\geq 10$ ). The meta-analysis [13] indicated that in the post-illness stage of SARS and MERS, the point prevalence of post-traumatic stress disorder was $32.2 \%$, that of depression was $14.9 \%$, and that of anxiety disorders was $14.8 \%$. However, our subjects did not include psychotic, confused, or cognitively disordered patients during admission.

In this study, the self-reported PTSD scale on admission was very useful in monitoring the mental status of COVID-19 patients in isolation wards.

Because of limited access to quarantined patients, early detection and rapid relief of psychiatric or behavioral problems might be particularly important [7]. These can be made possible by psychological tests or psychiatric consultations. Following admission, some patients suffered from later psychiatric symptoms, although their physical condition had become good. This is true because after the symptoms of COVID-19 have diminished, confirmed patients could not be discharged from the hospital until they had had two consecutive tests separated by 24 hours showing negative results. As these patients undergo this process, they are frequently apprehensive that the tests may become positive again [3]. This can generate a lot of anxiety because it can lead to a continuation of hospitalization. Our study found that self-reported PTSD during the initial period of admission was significantly predictive of psychiatric diagnoses at discharge. which was made by psychiatrists in spite of the possibilities of changes of patients' psychological status during whole period of admission. We did not find that self-reported depression on admission was associated with a psychiatric diagnosis or with the use of psychotropic medications.

Married subjects were at risk of being diagnosed with mental disorders in this study. They had more worries than single patients, which included worries about taking care of children, leaving home, spouses who may have also contracted COVID-19, various troublesome events occurring in families, or economic 
burdens suffered during hospitalization [14]. Many clusters of infection have been found within familial households. A study in China showed that the rate of secondary transmission among household contacts of patients with SARS-CoV-2 infection was 30\% [15]. Although we did not gather exact data, a moderate portion of married patients in our sample had spouses who had also contracted COVID-19. In addition, single subjects tended to endure psychological discomforts by themselves instead of getting help from the psychiatrists, fearing the stigma of mental illness.

In this study, being forty years old or older was one of the factors associated with the use of psychotropic agents. But this was not related to being diagnosed with psychiatric disorders. We observed that elderly patients tended to want to be prescribed medications when they felt anxiety or insomnia during restriction to hospital rooms. Young patients tried to cope with boredom and inactivity by free exercises, and they were reluctant to receive medications to aid sleep.

This study has several limitations. First, the sample used in this study consisted of medical records of admitted cases in a nationally designated COVID-19 hospital. Therefore, the results could not be generalized to all patients with COVID-19 undergoing treatment. Moreover, to make hospital beds available for severely ill COVID-19 patients, the South Korean government classifies confirmed patients and provides treatment in different settings, depending on the severity of their illness. Patients confirmed to be in severe conditions were admitted in specialized infectious disease hospitals (nationally designated treatment facilities). Patients who were asymptomatic or mildly symptomatic were provided medical services and monitoring in homes or facilities (Living and Treatment Centers) [10].

Second, self-reported psychiatric symptoms could either be underreported or overreported. Additionally, we excluded those subjects who had chronic psychotic disorders or who suffered confusion or dementia. So, in this study, we need to exercise caution as we interpret the prevalence of PTSD or symptoms of depression suffered during the acute phase. However, one purpose of this study was to determine if patient outcomes could be predicted by considering self-reported PTSD, psychotropic drug use, or a diagnosis of depression arrived at by psychiatrists assigned to the patients.

Despite these limitations, this study gives us insights into the mental health problems of COVID-19 patients, factors related to the psychiatric disorders, the use of psychotropic drugs offered in psychiatric consultations, and mental health services needed during the acute treatment phase in isolation wards. Previous studies of the mental health effects on MERS or SARS survivors reported that EIDs could cause sustained mental morbidity. Post-discharge follow-up studies might be needed to shed light on the longterm prognoses of the psychiatric problems that COVID-19 survivors experience.

\section{Abbreviations}

COVID-19

COronaVIrus Disease 2019

DSM-IV

Diagnostic and Statistical Manual of Mental disorders, 4th version 
DSM-5

Diagnostic and Statistical Manual of Mental disorders, 5th version

EID

Emerging Infectious Disease

IES-R-K

Impact of Event Scale-Revised-Korean

MERS

Middle East Respiratory Syndrome

NMC

National Medical Center

PCL-5

Posttraumatic Stress Disorder Checklist for the fifth edition of the Diagnostic and

\section{Declarations}

\section{Ethics approval and consent to participate}

The present study protocol was reviewed and approved by the Institutional Review Board for Human

Subjects at the NMC in Seoul, S. Korea (NMC-2006-005), and written informed consent was obtained from each patient.

\section{Consent for publication}

Not applicable

\section{Availability of data and materials}

The data that support the findings of this study are available from Ministry of Health \& Welfare of the Republic of Korea but restrictions apply to the availability of these data, which were used under license for the current study, and so are not publicly available. Data are however available from the authors upon reasonable request and with permission of Ministry of Health \& Welfare.

\section{Competing interest}

The authors declare that they have no competing interests.

\section{Funding}

This study was supported by a grant from the Korean Mental Health Technology R\&D Project, Ministry of Health \& Welfare, Republic of Korea (HL19C0007). 


\section{Acknowledgements}

Not applicable

\section{Authors' contributions}

JR Kim drafted the manuscript.

$\mathrm{MH}$ Han performed the data analysis and drafted the manuscript.

SH Lee conceived the study idea, and reviewed drafts of the manuscript.

All authors participated in interpreting results and approved the final manuscript.

\section{References}

1. An JY, Seo ER, Lim KH, Shin JH, Kim JB. Standardization of the Korean version of screening tool for depression (patient health Questionnaire-9, PHQ-9. J Korean Soc Biol Ther Psychiatry. 2013;19:4756.

2. Epstein D, Andrawis, et al. Anxiety and Suicidality in a Hospitalized Patient with COVID-19 Infection. European journal of case reports in internal medicine. 2020;7(5):001651. https://doi.org/10.12890/2020_001651.

3. Grover S, Dua D, Sahoo S, Mehra A, Nehra R, Chakrabarti S. Why all COVID-19 hospitals should have mental health professionals: The importance of mental health in a worldwide crisis! Asian journal of psychiatry. 2020;51:102147. https://doi.org/10.1016/j.ajp.2020.102147. Advance online publication.

4. Jae HS, Hee CK, Chul HJ, et al. The Standardization of the Korean Version of the Patient Health Questionnaire-2. J Korean Neuropsychiatr Assoc. 2013;52:115-21.

5. James PB, Wardle J, Steel A, Adams J. Post-Ebola psychosocial experiences and coping mechanisms among Ebola survivors: a systematic review. Trop Med Int Health. 2019;24(6):671-91. doi:10.1111/tmi.13226.

6. Jung YE, Kim D, Kim WH, Roh D, Chae JH, Park JE. A Brief Screening Tool for PTSD: Validation of the Korean Version of the Primary Care PTSD Screen for DSM-5 (K-PC-PTSD-5). J Korean Med Sci. 2018; $33(52)$.

7. Kim HC, Yoo SY, Lee BH, Lee SH, Shin HS. Psychiatric Findings in Suspected and Confirmed Middle East Respiratory Syndrome Patients Quarantined in Hospital: A Retrospective Chart Analysis.

Psychiatry Investig. 2018;15(4):355-60. doi:10.30773/pi.2017.10.25.1.

8. Kroenke K, Spitzer RL, Williams JBW. The PHQ-9: validity of a brief depression severity measure. J Gen Intern Med. 2001;16(9):606-13.

9. Leung GM, Hedley, et al. The epidemiology of severe acute respiratory syndrome in the 2003 Hong Kong epidemic: an analysis of all 1755 patients. Ann Intern Med. 2004;141(9):662-73. 
10. Ministry of Health and Welfare. Coronavirus Disease-19: cases in Korea. http://ncov.mohw.go.kr/en. Accessed 7 June 2020.

11. Park HY, Park WB, Lee SH, et al. Posttraumatic stress disorder and depression of survivors 12 months after the outbreak of Middle East respiratory syndrome in South Korea. BMC Public Health. 2020;20(1):605. doi:10.1186/s12889-020-08726-1.

12. Qi R, Chen, et al. Psychological morbidities and fatigue in patients with confirmed COVID-19 during disease outbreak: prevalence and associated biopsychosocial risk factors. medRxiv. 2020. https://doi.org/10.1101/2020.05.08.20031666.

13. Rogers JP, Chesney E, Oliver D, et al. Psychiatric and neuropsychiatric presentations associated with severe coronavirus infections: a systematic review and meta-analysis with comparison to the COVID19 pandemic [published online ahead of print, 2020 May 18]. Lancet Psychiatry. 2020; S22150366(20)30203-0. doi:10.1016/S2215-0366(20)30203-0.

14. Sahoo S, Mehra, et al.Lived experiences of the corona survivors (patients admitted in COVID wards): A narrative real-life documented summaries of internalized guilt, shame, stigma, anger. Asian journal of psychiatry $2020 ; 53,102187$. Advance online publication. https://doi.org/10.1016/j.ajp.2020.102187.

15. Wang Z, Ma W, Zheng X, Wu G, Zhang R. Household transmission of SARS-CoV-2 [published online ahead of print, 2020 Apr 10]. J Infect. 2020;81(1):179-82. doi:10.1016/j.jinf.2020.03.040.

16. Weathers FW, Litz BT, Keane TM, Palmieri PA, Marx BP, Schnurr PP. The PTSD Checklist for DSM-5 (PCL-5). 2013. Available from: http://www.ptsd.va.gov.

17. World Health Organization. Coronavirus infections: disease outbreak news. World Health Organization. http://www.who.int/csr/don/26-april-2016-mers-saudi-arabia/en/. Accessed 7 June 2020.

18. Xiang YT, Yang Y, Li W, Zhang L, Zhang Q, Cheung T, Ng CH. Timely mental health care for the 2019 novel coronavirus outbreak is urgently needed. Lancet Psychiatry. 2020;7(3):228-9.

19. Yuan B, Li, et al. Correlation between immune response and self-reported depression during convalescence from COVID-19, Brain, Behavior, and Immunity. 2020; doi: https://doi.org/10.1016/j.bbi.2020.05.062.

20. Zhou F. Clinical course and risk factors for mortality of adult inpatients with COVID-19 in Wuhan, China: a retrospective cohort study. Lancet. 2020. 